\title{
On Periodic Solutions of Higher-Order Functional Differential Equations
}

\author{
I. Kiguradze, ${ }^{1}$ N. Partsvania, ${ }^{1}$ and B. Půža ${ }^{2}$ \\ ${ }^{1}$ Andrea Razmadze Mathematical Institute, 1 Aleksidze Street, 0193 Tbilisi, Georgia \\ ${ }^{2}$ Department of Mathematics and Statistics, Masaryk University, Janáckovo nám. $2 a$, \\ 66295 Brno, Czech Republic
}

Correspondence should be addressed to I. Kiguradze, kig@rmi.acnet.ge

Received 8 September 2007; Accepted 23 January 2008

Recommended by Donal O'Regan

For higher-order functional differential equations and, particularly, for nonautonomous differential equations with deviated arguments, new sufficient conditions for the existence and uniqueness of a periodic solution are established.

Copyright ( 2008 I. Kiguradze et al. This is an open access article distributed under the Creative Commons Attribution License, which permits unrestricted use, distribution, and reproduction in any medium, provided the original work is properly cited.

\section{Statement of the main results}

\subsection{Statement of the problem}

Let $n \geq 2$ be a natural number, $\omega>0, L_{\omega}$ the space of $\omega$-periodic and Lebesgue integrable on $[0, \omega]$ functions $u: \mathbb{R} \rightarrow \mathbb{R}$ with the norm

$$
\|u\|_{L_{\omega}}=\int_{0}^{\omega}|u(s)| d s .
$$

Let $C_{\omega}$ and $C_{\omega}^{n-1}$ be, respectively, the spaces of continuous and $(n-1)$-times continuously differentiable $\omega$-periodic functions with the norms

$$
\|u\|_{C_{\omega}}=\max \{|u(t)|: t \in \mathbb{R}\}, \quad\|u\|_{C_{\omega}^{n-1}}=\sum_{k=1}^{n}\left\|u^{(k-1)}\right\|_{C_{\omega}}
$$

and let $\widetilde{C}_{\omega}^{n-1}$ be the space of functions $u \in C_{\omega}^{n-1}$ for which $u^{(n-1)}$ is absolutely continuous. 
We consider the functional differential equation

$$
u^{(n)}(t)=f(u)(t),
$$

whose important particular case is the differential equation with deviated arguments

$$
u^{(n)}(t)=g\left(t, u\left(\tau_{1}(t)\right), \ldots, u^{(n-1)}\left(\tau_{n}(t)\right)\right)
$$

Throughout the paper, it is assumed that $f: C_{\omega}^{n-1} \rightarrow L_{\omega}$ is a continuous operator satisfying the condition

$$
f_{r}^{*}(\cdot)=\sup \{|f(u)(\cdot)|:\|u\| \leq r\} \in L_{\omega} \quad \text { for any } r>0,
$$

and $g: \mathbb{R} \times \mathbb{R}^{n} \rightarrow \mathbb{R}$ is a function from the Carathéodory class, satisfying the equality

$$
g\left(t+\omega, x_{1}, \ldots, x_{n}\right)=g\left(t, x_{1}, \ldots, x_{n}\right)
$$

for almost all $t \in \mathbb{R}$ and all $\left(x_{1}, \ldots, x_{n}\right) \in \mathbb{R}^{n}$. As for the functions $\tau_{k}: \mathbb{R} \rightarrow \mathbb{R}(k=1, \ldots, n)$, they are measurable on each finite interval and

$$
\frac{\left(\tau_{k}(t+\omega)-\tau_{k}(t)\right)}{\omega} \text { is an integer }(k=1, \ldots, n)
$$

for almost all $t \in \mathbb{R}$.

A function $u \in \widetilde{C}_{\omega}^{n-1}$ is said to be an $\omega$-periodic solution of (1.3) or (1.4) if it satisfies this equation almost everywhere on $\mathbb{R}$.

For the case $\tau_{k}(t) \equiv t(k=1, \ldots, n)$, the problem on the existence and uniqueness of an $\omega$-periodic solution of (1.4) has been investigated in detail (see, e.g., [1-18] and the references therein). For (1.3) and (1.4), where $\tau_{k}(t) \not \equiv t(k=1, \ldots, n)$, the mentioned problem is studied mainly in the cases $n \in\{1,2\}$ (see [19-31]), and for the case $n>2$, the problem remains so far unstudied. The present paper is devoted exactly to this case.

Everywhere below the following notation will be used:

$$
\begin{gathered}
v_{k}=\frac{\omega}{2}\left(\frac{\omega}{2 \pi}\right)^{n-k-2} \quad(k=0, \ldots, n-2), \quad v_{n-1}=1, \\
{[x]_{-}=(|x|-x) / 2 \text { for } x \in \mathbb{R},} \\
\mu(u)=\min \{|u(t)|: 0 \leq t \leq \omega\} \quad \text { for } u \in C_{\omega} .
\end{gathered}
$$

\subsection{Existence theorems}

The existence of an $\omega$-periodic solution of (1.3) is proved in the cases where the operator $f$ in the space $C_{\omega}^{n-1}$ satisfies the conditions

$$
\begin{gathered}
\left(\int_{0}^{\omega} f(u)(s) d s\right) \operatorname{sgn}(\sigma u(0)) \geq h(\mu(u))-\sum_{k=1}^{n-1} e_{1 k}\left\|u^{(k)}\right\|_{C_{\omega}}-c \quad \text { for } \mu(u)>0, \\
\left|\int_{t}^{x} f(u)(s) d s\right| \leq \ell h(\mu(u))+\sum_{k=1}^{n-1} \ell_{2 k}\left\|u^{(k)}\right\|_{C_{\omega}}+c \quad \text { for } 0 \leq t \leq x \leq \omega,
\end{gathered}
$$


I. Kiguradze et al.

or the conditions

$$
\begin{gathered}
\left(\int_{0}^{\omega} f(u)(s) d s\right) \operatorname{sgn}(\sigma u(0)) \geq 0 \quad \text { for } \mu(u)>c_{0}, \\
\left|\int_{t}^{x} f(u)(s) d s\right| \leq c_{0}+\sum_{k=0}^{n-1} \ell_{k}\left\|u^{(k)}\right\|_{C_{\omega}} \quad \text { for } 0 \leq t \leq x \leq \omega .
\end{gathered}
$$

Theorem 1.1. Let there exist an increasing function $h:[0,+\infty[\rightarrow[0,+\infty[$ and constants $c \geq 0$, $\ell_{i k} \geq 0(i=1,2 ; k=1, \ldots, n-1), \ell \geq 1$, and $\sigma \in\{-1,1\}$ such that $h(x) \rightarrow+\infty$ as $x \rightarrow+\infty$,

$$
\sum_{k=1}^{n-1}\left(\ell \ell_{1 k}+\ell_{2 k}\right) v_{k}<1
$$

and inequalities (1.11) and (1.12) are satisfied in the space $C_{\omega}^{n-1}$. Then (1.3) has at least one w-periodic solution.

Theorem 1.2. Let there exist constants $c_{0} \geq 0, \ell_{k} \geq 0(k=0, \ldots, n-1)$, and $\sigma \in\{-1,1\}$ such that

$$
\sum_{k=0}^{n-1} e_{k} v_{k}<1
$$

and inequalities (1.13) and (1.14) are satisfied in the space $C_{\omega}^{n-1}$. Then (1.3) has at least one w-periodic solution.

Theorems 1.1 and 1.2 imply the following propositions.

Corollary 1.3. Let there exist constants $\lambda>0, \sigma \in\{-1,1\}$, and functions $p_{i k} \in L_{\omega}(i, k=1, \ldots, n)$, $q \in L_{\omega}$ such that the inequalities

$$
\begin{gathered}
g\left(t, x_{1}, \ldots, x_{n}\right) \operatorname{sgn}\left(\sigma x_{1}\right) \geq p_{11}(t)\left|x_{1}\right|^{\lambda}-\sum_{k=2}^{n} p_{1 k}(t)\left|x_{k}\right|-q(t), \\
\left|g\left(t, x_{1}, \ldots, x_{n}\right)\right| \leq p_{21}(t)\left|x_{1}\right|^{\lambda}+\sum_{k=2}^{n} p_{2 k}(t)\left|x_{k}\right|+q(t)
\end{gathered}
$$

hold on the set $\mathbb{R} \times \mathbb{R}^{n}$. Let, moreover,

$$
\int_{0}^{\omega} p_{11}(t) d t>0
$$

and either $\lambda<1$ and

$$
\sum_{k=2}^{n} v_{k-1} \int_{0}^{\omega}\left(\ell p_{1 k}(s)+p_{2 k}(s)\right) d s<1
$$

or $\lambda=1$ and

$$
v_{0} \int_{0}^{\omega}\left(\ell\left[p_{11}(s)\right]_{-}+p_{21}(s)\right) d s+\sum_{k=2}^{n} v_{k-1} \int_{0}^{\omega}\left(\ell p_{1 k}(s)+p_{2 k}(s)\right) d s<1,
$$

where $\ell=\int_{0}^{\omega} p_{21}(t) d t / \int_{0}^{\omega} p_{11}(t) d t$. Then (1.4) has at least one w-periodic solution. 
Corollary 1.4. Let there exist constants $c_{0} \geq 0, \sigma \in\{-1,1\}$, and functions $g_{0} \in L_{\omega}, p_{k} \in L_{\omega}(k=$ $1, \ldots, n), q \in L_{\omega}$ such that

$$
\int_{0}^{\omega} g_{0}(s) d s=0
$$

and the inequalities

$$
\begin{gathered}
\left(g\left(t, x_{1}, \ldots, x_{n}\right)-g_{0}(t)\right) \operatorname{sgn}\left(\sigma x_{1}\right) \geq 0 \text { for }\left|x_{1}\right|>c_{0} \\
\left|g\left(t, x_{1}, \ldots, x_{n}\right)\right| \leq \sum_{k=1}^{n} p_{k}(t)\left|x_{k}\right|+q(t)
\end{gathered}
$$

hold on the set $\mathbb{R} \times \mathbb{R}^{n}$. If, moreover,

$$
\sum_{k=1}^{n} v_{k-1} \int_{0}^{\omega} p_{k}(s) d s<1
$$

then (1.4) has at least one w-periodic solution.

\subsection{Uniqueness theorems}

The unique solvability of a periodic problem for (1.3) is proved in the cases where the operator $f$, for any $u$ and $v \in C_{\omega}^{n-1}$, satisfies the conditions:

$$
\begin{gathered}
\left(\int_{0}^{\omega}(f(u+v)(s)-f(v)(s)) d s\right) \operatorname{sgn}(\sigma u(0)) \geq \ell_{10} \mu(u)-\sum_{k=1}^{n-1} \ell_{1 k}\left\|u^{(k)}\right\|_{C_{\omega}} \quad \text { for } \mu(u)>0, \\
\quad\left|\int_{t}^{x}(f(u+v)(s)-f(v)(s)) d s\right| \leq \ell_{20} \mu(u)+\sum_{k=1}^{n-1} \ell_{2 k}\left\|u^{(k)}\right\|_{C_{\omega}} \text { for } 0 \leq t \leq x \leq \omega,
\end{gathered}
$$

or the conditions

$$
\begin{aligned}
& \left(\int_{0}^{\omega}(f(u+v)(s)-f(v)(s)) d s\right) \operatorname{sgn}(\sigma u(0))>0 \text { for } \mu(u)>0, \\
& \left|\int_{t}^{x}(f(u+v)(s)-f(v)(s)) d s\right| \leq \ell_{0}\|u\|_{C_{\omega}} \text { for } 0 \leq t \leq x \leq \omega .
\end{aligned}
$$

Theorem 1.5. Let there exist constants $\ell_{20} \geq \ell_{10}>0, \ell_{i k} \geq 0(i=1,2 ; k=1, \ldots, n-1)$, and $\sigma \in\{-1,1\}$ such that for arbitrary $u, v \in C_{\omega}^{n-1}$ the operator $f$ satisfies inequalities (1.24) and (1.25). If, moreover, inequality (1.15) holds, where $\ell=\ell_{20} / \ell_{10}$, then (1.3) has one and only one w-periodic solution.

Theorem 1.6. Let there exist constants $\ell_{0}>0$ and $\sigma \in\{-1,1\}$ such that for arbitrary $u, v \in C_{\omega}^{n-1}$ an operator $f$ satisfies conditions (1.26) and (1.27). If, moreover,

$$
\int_{0}^{\omega} f(0)(s) d s=0, \quad \ell_{0} v_{0}<1
$$

then (1.3) has one and only one w-periodic solution. 
From Theorem 1.5, the following corollary holds.

Corollary 1.7. Let there exist a constant $\sigma \in\{-1,1\}$ and functions $p_{i k} \in L_{\omega}(i=1,2 ; k=1, \ldots, n)$ such that for almost all $t \in \mathbb{R}$ and all $\left(x_{1}, \ldots, x_{n}\right)$ and $\left(y_{1}, \ldots, y_{n}\right) \in \mathbb{R}^{n}$ the conditions

$$
\begin{gathered}
\left(g\left(t, x_{1}, \ldots, x_{n}\right)-g\left(t, y_{1}, \ldots, y_{n}\right)\right) \operatorname{sgn}\left(\sigma\left(x_{1}-y_{1}\right)\right) \geq p_{11}(t)\left|x_{1}-y_{1}\right|-\sum_{k=2}^{n} p_{1 k}(t)\left|x_{k}-y_{k}\right| \\
\left|g\left(t, x_{1}, \ldots, x_{n}\right)-g\left(t, y_{1}, \ldots, y_{n}\right)\right| \leq \sum_{k=1}^{n} p_{2 k}(t)\left|x_{k}-y_{k}\right|
\end{gathered}
$$

are satisfied. If, moreover, inequalities (1.18) and (1.20) hold, where $\ell=\int_{0}^{\omega} p_{21}(s) d s / \int_{0}^{\omega} p_{11}(s) d s$, then (1.4) has one and only one w-periodic solution.

Note that the functions $p_{1 k}(k=2, \ldots, n)$ and $p_{2 k}(k=1, \ldots, n)$ in this corollary as in Corollary 1.3 are nonnegative, and $p_{11}$ may change its sign.

Consider now the equation

$$
u^{(n)}(t)=g(t, u(\tau(t)))
$$

which is derived from (1.4) in the case where $g\left(t, x_{1}, \ldots, x_{n}\right) \equiv g\left(t, x_{1}\right)$ and $\tau_{1}(t) \equiv \tau(t)$. As above, we will assume that the function $g: \mathbb{R} \times \mathbb{R} \rightarrow \mathbb{R}$ belongs to the Carathéodory class and

$$
g(t+\omega, x)=g(t, x)
$$

for almost all $t \in \mathbb{R}$ and all $x \in \mathbb{R}$. As for the function $\tau: \mathbb{R} \rightarrow \mathbb{R}$, it is measurable on each finite interval and

$$
\frac{(\tau(t+\omega)-\tau(t))}{\omega} \text { is an integer }
$$

for almost all $t \in \mathbb{R}$.

Theorem 1.6 yields the following corollary.

Corollary 1.8. Let there exist a constant $\sigma \in\{-1,1\}$ and a function $p \in L_{\omega}$ such that the condition

$$
0<(g(t, x)-g(t, y)) \operatorname{sgn}(\sigma(x-y)) \leq p(t)|x-y|
$$

holds for almost all $t \in \mathbb{R}$ and all $x \neq y$. If, moreover,

$$
\int_{0}^{\omega} g(s, 0) d s=0, \quad v_{0} \int_{0}^{\omega} p(s) d s<1,
$$

then (1.30) has one and only one w-periodic solution. 


\section{Auxiliary propositions}

\subsection{Lemmas on a priori estimates}

Everywhere in this section, we will assume that $v_{k}(k=0, \ldots, n-1)$ are numbers given by (1.13).

Lemma 2.1. If $u \in C_{\omega}^{n-1}$, then

$$
\begin{gathered}
\|u\|_{C_{\omega}} \leq \mu(u)+v_{0}\left\|u^{(n-1)}\right\|_{C_{\omega^{\prime}}} \\
\left\|u^{(k)}\right\|_{C_{\omega}} \leq v_{k}\left\|u^{(n-1)}\right\|_{C_{\omega}} \quad(k=1, \ldots, n-1) .
\end{gathered}
$$

Proof. We choose $t_{0} \in[0, \omega]$ so that

$$
u\left(t_{0}\right)=\mu(u),
$$

and suppose

$$
v(t)=u(t)-u\left(t_{0}\right)
$$

Then $v\left(t_{0}\right)=v\left(t_{0}+\omega\right)=0$. Thus

$$
|v(t)|=\left|\int_{t_{0}}^{t} v^{\prime}(s) d s\right| \leq \int_{t_{0}}^{t}\left|v^{\prime}(s)\right| d s, \quad|v(t)|=\left|\int_{t}^{t_{0}+\omega} v^{\prime}(s) d s\right| \leq \int_{t}^{t_{0}+\omega}\left|v^{\prime}(s)\right| d s \quad \text { for } 0 \leq t \leq \omega .
$$

If we sum up these two inequalities, we obtain

$$
2|v(t)| \leq \int_{t_{0}}^{t_{0}+\omega}\left|v^{\prime}(s)\right| d s \quad \text { for } 0 \leq t \leq \omega
$$

Consequently,

$$
\|v\|_{C_{\omega}} \leq \frac{1}{2} \int_{t_{0}}^{t_{0}+\omega}\left|v^{\prime}(s)\right| d s
$$

However,

$$
\|u\|_{C_{\omega}} \leq \mu(u)+\|v\|_{C_{\omega}}, \quad \int_{t_{0}}^{t_{0}+\omega}\left|v^{\prime}(s)\right| d s=\int_{0}^{\omega}\left|u^{\prime}(s)\right| d s,
$$

which together with the previous inequality yields

$$
\|u\|_{C_{\omega}} \leq \mu(u)+\frac{1}{2} \int_{0}^{\omega}\left|u^{\prime}(s)\right| d s \leq \mu(u)+\frac{1}{2} \omega^{1 / 2}\left(\int_{0}^{\omega}\left|u^{\prime}(s)\right|^{2} d s\right)^{1 / 2} .
$$

On the other hand, by the Wirtinger inequality (see [32, Theorem 258] and [13, Lemma 1.1]), we have

$$
\int_{0}^{\omega}\left|u^{\prime}(s)\right|^{2} d s \leq\left(\frac{\omega}{2 \pi}\right)^{2 n-4} \int_{0}^{\omega}\left|u^{(n-1)}(s)\right|^{2} d s \leq \omega\left(\frac{\omega}{2 \pi}\right)^{2 n-4}\left\|u^{(n-1)}\right\|_{C_{\omega}}^{2} .
$$

Consequently, estimate (2.1) is valid. 
If now we take into account that $u^{(k)} \in C_{\omega}^{n-1-k}$ and $\mu\left(u^{(k)}\right)=0(k=1, \ldots, m)$, then the validity of estimates (2.2) becomes evident.

Lemma 2.2. Let $u \in C_{\omega}^{n-1}$ and

$$
\left\|u^{(n-1)}\right\|_{C_{\omega}} \leq c_{0}+\sum_{k=0}^{n-1} \ell_{k}\left\|u^{(k)}\right\|_{C_{\omega^{\prime}}}
$$

where $c_{0}$ and $\ell_{k}(k=0, \ldots, n-1)$ are nonnegative constants. If, moreover,

$$
\delta=\sum_{k=0}^{n-1} \ell_{k} v_{k}<1
$$

then

$$
\begin{gathered}
\left\|u^{(n-1)}\right\|_{C_{\omega}} \leq(1-\delta)^{-1}\left(c_{0}+\ell_{0} \mu(u)\right) \\
\|u\|_{C_{\omega}^{n-1}} \leq \mu(u)+(1-\delta)^{-1}\left(c_{0}+\ell_{0} \mu(u)\right) \sum_{k=0}^{n-1} v_{k} .
\end{gathered}
$$

Proof. By Lemma 2.1, the function $u$ satisfies inequalities (2.1) and (2.2). In view of these inequalities from (2.11) we find

$$
\left\|u^{(n-1)}\right\|_{C_{\omega}} \leq c_{0}+\ell_{0} \mu(u)+\left(\sum_{k=0}^{n-1} \ell_{k} v_{k}\right)\left\|u^{(n-1)}\right\|_{C_{\omega}} .
$$

Hence, by virtue of condition (2.12), we have estimate (2.13). On the other hand, according to (2.13), inequalities (2.1) and (2.2) result in (2.14).

Lemma 2.3. Let $u \in C_{\omega}^{n-1}$ and

$$
\mu(u) \leq \varphi\left(\left\|u^{(n-1)}\right\|_{C_{\omega}}\right), \quad\left\|u^{(n-1)}\right\|_{C_{\omega}} \leq c_{0}+\sum_{k=1}^{n-1} \ell_{k}\left\|u^{(k)}\right\|_{C_{\omega^{\prime}}}
$$

where $\varphi:\left[0,+\infty\left[\rightarrow\left[0,+\infty\left[\right.\right.\right.\right.$ is a nondecreasing function, $c_{0} \geq 0, \ell_{k} \geq 0(k=1, \ldots, n-1)$, and

$$
\delta=\sum_{k=1}^{n-1} e_{k} v_{k}<1
$$

Then

$$
\|u\|_{C_{\omega}^{n-1}} \leq r_{0}
$$

where

$$
r_{0}=\varphi\left((1-\delta)^{-1} c_{0}\right)+(1-\delta)^{-1} c_{0} \sum_{k=0}^{n-1} v_{k} .
$$

Proof. Inequalities (2.16) and (2.17) imply inequalities (2.11) and (2.12), where $\ell_{0}=0$. However, by Lemma 2.2 , these inequalities guarantee the validity of the estimates

$$
\left\|u^{(n-1)}\right\|_{C_{\omega}} \leq(1-\delta)^{-1} c_{0}, \quad\|u\|_{C_{\omega}^{n-1}} \leq \mu(u)+(1-\delta)^{-1} c_{0} \sum_{k=0}^{n-1} v_{k} .
$$


On the other hand, according to the first inequality in (2.16), we have

$$
\mu(u) \leq \varphi\left((1-\delta)^{-1} c_{0}\right)
$$

Consequently, estimate (2.18) is valid, where $r_{0}$ is a number given by equality (2.19).

Analogously, from Lemma 2.2, the following hold.

Lemma 2.4. Let $u \in C_{\omega}^{n-1}$ and

$$
\mu(u) \leq c_{0}, \quad\left\|u^{(n-1)}\right\|_{C_{\omega}} \leq c_{0}+\sum_{k=0}^{n-1} \ell_{k}\left\|u^{(k)}\right\|_{C_{\omega^{\prime}}}
$$

where $c_{0} \geq 0, \ell_{k} \geq 0(k=0, \ldots, n-1)$. If, moreover, inequality (2.12) holds, then estimate (2.18) is valid, where

$$
r_{0}=\left[1+(1-\delta)^{-1}\left(1+\ell_{0}\right) \sum_{k=0}^{n-1} v_{k}\right] c_{0}
$$

\subsection{Lemma on the solvability of a periodic problem}

Below, by $C^{n-1}([0, \omega])$ we denote the space of $(n-1)$-times continuously differentiable functions $u:[0, \omega] \rightarrow \mathbb{R}$ with the norm

$$
\|u\|_{C^{n-1}([0, \omega])}=\sum_{k=1}^{n} \max \left\{\left|u^{(k-1)}(t)\right|: 0 \leq t \leq \omega\right\},
$$

and by $L([0, \omega])$ we denote the space of Lebesgue integrable functions $u:[0, \omega] \rightarrow \mathbb{R}$ with the norm

$$
\|u\|_{L([0, \omega])}=\int_{0}^{\omega}|u(t)| d t
$$

Consider the differential equation

$$
u^{(n)}(t)=\bar{f}(u)(t)
$$

with the periodic boundary conditions

$$
u^{(i-1)}(0)=u^{(i-1)}(\omega) \quad(i=1, \ldots, n),
$$

where $\bar{f}: C^{n-1}([0, \omega]) \rightarrow L([0, \omega])$ is a continuous operator such that

$$
\bar{f}_{r}(\cdot)=\sup \left\{|\bar{f}(u)(\cdot)|:\|u\|_{C^{n-1}([0, \omega])} \leq r\right\} \in L([0, \omega])
$$

for any $r>0$. The following lemma is valid. 
Lemma 2.5. Let there exist a linear, bounded operator $p: C^{n-1}([0, \omega]) \rightarrow L([0, \omega])$ and a positive constant $r_{0}$ such that the linear differential equation

$$
u^{(n)}(t)=p(u)(t)
$$

with the periodic conditions (2.27) has only a trivial solution and for an arbitrary $\lambda \in] 0,1[$ every solution of the differential equation

$$
u^{(n)}(t)=\lambda p(u)(t)+(1-\lambda) f(u)(t),
$$

satisfying condition (2.27), admits the estimate

$$
\|u\|_{C^{n-1}([0, \omega])} \leq r_{0} .
$$

Then problem (2.26), (2.27) has at least one solution.

For the proof of this lemma see [33, Corollary 2].

Lemma 2.6. Let $f: C_{\omega}^{n-1} \rightarrow L_{\omega}$ be a continuous operator satisfying condition (1.5) for any $r>0$. Let, moreover, there exist constants $a \neq 0$ and $r_{0}>0$ such that for an arbitrary $\left.\lambda \in\right] 0,1[$, every w-periodic solution of the functional differential equation

$$
u^{(n)}(t)=\lambda a u(0)+(1-\lambda) f(u)(t)
$$

admits estimate (2.18). Then (1.3) has at least one w-periodic solution.

Proof. Let $c_{1}, \ldots, c_{n}$ be arbitrary constants. Then the problem

$$
y^{(2 n)}(t)=0, \quad y^{(i-1)}(0)=0, \quad y^{(i-1)}(\omega)=c_{i} \quad(i=1, \ldots, n)
$$

has a unique solution. Let us denote by $y\left(t ; c_{1}, \ldots, c_{n}\right)$ the solution of that problem.

For any $u \in C^{n-1}([0, \omega])$, we set

$$
z(u)(t)=u(t)-y\left(t ; u(\omega)-u(0), \ldots, u^{(n-1)}(\omega)-u^{(n-1)}(0)\right) \text { for } 0 \leq t \leq \omega,
$$

and extend $z(u)(\cdot)$ to $\mathbb{R}$ periodically with a period $\omega$. Then, it is obvious that $z: C^{n-1}([0, \omega]) \rightarrow$ $C_{\omega}^{n-1}$ is a linear, bounded operator.

Suppose

$$
\bar{f}(u)(t)=f(z(u))(t) .
$$

Consider the boundary value problem (2.26), (2.27). If the function $u$ is an $\omega$-periodic solution of (1.3), then its restriction to $[0, \omega]$ is a solution of problem $(2.26),(2.27)$, and vice versa, if $u$ is a solution of problem (2.26), (2.27), then its periodic extension to $\mathbb{R}$ with a period $\omega$ is an $\omega$-periodic solution of (1.3). Thus to prove the lemma, it suffices to state that problem (2.26), (2.27) has at least one solution.

By virtue of equalities (2.34), (2.35) and condition $(1.5), \bar{f}: C^{n-1}([0, \omega]) \rightarrow L([0, \omega])$ is a continuous operator, satisfying condition (2.28) for any $r>0$. On the other hand, it is evident that if $p(u)(t) \equiv \alpha u(0)$, then problem (2.29), (2.27) has only a trivial solution. By these conditions and Lemma 2.5, problem (2.26), (2.27) is solvable if for any $\lambda \in] 0,1$ [ every solution $u$ of problem (2.30), (2.27), where $p(u)(t) \equiv \alpha u(0)$, admits estimate (2.31).

Let $u$ be a solution of problem (2.30), (2.27) for some $\lambda \in] 0,1[$. Then its periodic extension to $\mathbb{R}$ with a period $\omega$ is a solution of (2.32), and according to one of the conditions of the lemma, admits estimate (2.18). Therefore, estimate (2.31) is valid. 


\section{Proof of the main results}

Proof of Theorem 1.1. Without loss of generality, it can be assumed that $h(0)=0$. On the other hand, according to condition (1.15), we can choose a constant $a$ so that $\sigma a>0$ and the numbers

$$
\ell_{k}=\ell_{1 k}+\ell \ell_{2 k} \quad(k=1, \ldots, n-2), \quad \ell_{n-1}=\ell_{1 n-1}+\ell \ell_{2 n-1}+\omega v_{0}|a|
$$

satisfy inequality (2.17).

Let

$$
h_{0}(x)=\min \{|a| \omega x, h(x)\},
$$

let $h_{0}^{-1}$ be a function, inverse to $h_{0}$,

$$
\varphi(x)=h_{0}^{-1}\left(\left(\sum_{k=1}^{n-1} \ell_{1 k} v_{k}\right) x+c\right), \quad c_{0}=2 c,
$$

and let $r_{0}$ be a number given by equality (2.19). By virtue of Lemma 2.6, to prove the theorem, it suffices to state that for any $\lambda \in] 0,1$ [ every $\omega$-periodic solution of (2.32) admits estimate (2.18).

Due to condition (1.12), from (2.32), we find

$$
\begin{aligned}
\left\|u^{(n-1)}\right\|_{C_{\omega}} & \leq \max \left\{\left|\int_{t}^{x} u^{(n)}(s) d s\right|: 0 \leq t \leq x \leq \omega\right\} \\
& \leq \lambda \omega|a||u(0)|+(1-\lambda) \operatorname{lh}(\mu(u))+\sum_{k=1}^{n-1} \ell_{2 k}\left\|u^{(k)}\right\|_{C_{\omega}}+c .
\end{aligned}
$$

On the other hand, if $\mu(u)>0$, then by condition (1.11) we have

$$
0=\left(\int_{0}^{w} u^{(n)}(s) d s\right) \operatorname{sgn}(\sigma u(0)) \geq \lambda \omega|a||u(0)|+(1-\lambda) h(\mu(u))-\sum_{k=1}^{n-1} \ell_{1 k}\left\|u^{(k)}\right\|_{C_{\omega}}-c,
$$

and consequently,

$$
\lambda \omega|a||u(0)|+(1-\lambda) h(\mu(u)) \leq \sum_{k=1}^{n-1} \ell_{1 k}\left\|u^{(k)}\right\|_{C_{\omega}}+c
$$

If $\mu(u)>0$, then by Lemma 2.1 and notations (3.1)-(3.3), from (3.4) and (3.6), inequalities (2.16) hold. And if $\mu(u)=0$, then by Lemma 2.1,

$$
|u(0)| \leq v_{0}\left\|u^{(n-1)}\right\|_{C_{\omega}} .
$$

On the other hand, $h(\mu(u))=h(0)=0$. Thus from (3.4) we obtain

$$
\left\|u^{(n-1)}\right\|_{C_{\omega}} \leq \omega v_{0}|a|\left\|u^{(n-1)}\right\|_{C_{\omega}}+\sum_{k=1}^{n-1} \ell_{2 k}\left\|u^{(k)}\right\|_{C_{\omega}}+c
$$


If along with this we take into account notations (3.1) and (3.3), then it becomes evident that inequalities (2.16) are fulfilled also in the case $\mu(u)=0$.

However, by Lemma 2.3, inequalities (2.16) and (2.17) guarantee the validity of estimate (2.18).

Proof of Theorem 1.2. Due to (1.16), inequality (2.12) holds. Let $r_{0}$ be a number given by equality (2.23) and

$$
a=\frac{\left(\sigma \ell_{0}\right)}{\omega}
$$

By Lemma 2.6, to prove the theorem, it suffices to state that for any $\lambda \in] 0,1[$ every $\omega$-periodic solution $u$ of (2.32) admits estimate (2.18).

If we suppose

$$
\mu(u)>c_{0}
$$

then in view of (1.13) and (3.9) from (2.32) we find

$$
0=\left(\int_{0}^{w} u^{(n)}(s) d s\right) \operatorname{sgn}(\sigma u(0))=\lambda \ell_{0}|u(0)|+(1-\lambda)\left(\int_{0}^{w} f(u)(s) d s\right) \operatorname{sgn}(\sigma u(0))>0 .
$$

The contradiction obtained proves that

$$
\mu(u) \leq c_{0} .
$$

On the other hand, according to (1.14) and (3.9), we have

$$
\begin{aligned}
\left\|u^{(n-1)}\right\|_{C_{\omega}} & \leq \max \left\{\left|\int_{t}^{x} u^{(n)}(s) d s\right|: 0 \leq t \leq x \leq \omega\right\} \\
& \leq \lambda \ell_{0}|u(0)|+(1-\lambda) \sum_{k=0}^{n-1} \ell_{k}\left\|u^{(k)}\right\|_{C_{\omega}}+c_{0} \leq \sum_{k=0}^{n-1} \ell_{k}\left\|u^{(k)}\right\|_{C_{\omega}}+c_{0} .
\end{aligned}
$$

Therefore, inequalities (2.22) are satisfied. However, by Lemma 2.4, inequalities (2.12) and (2.22) guarantee the validity of estimate (2.18).

Proof of Corollary 1.3. For an arbitrary $u \in C_{\omega}^{n-1}$, we set

$$
f(u)(t)=g\left(t, u\left(\tau_{1}(t)\right), \ldots, u^{(n-1)}\left(\tau_{n}(t)\right)\right) .
$$

Then (1.4) takes the form (1.3). It is obvious that $f: C_{\omega}^{n-1} \rightarrow L_{\omega}$ is a continuous operator, satisfying condition (1.8), since $g: \mathbb{R} \times \mathbb{R}^{n} \rightarrow \mathbb{R}$ belongs to the Carathéodory class and conditions (1.6) and (1.7) are satisfied.

Let $u \in C_{\omega}^{n-1}$ and $\mu(u)=u\left(t_{0}\right)$. Then by Lemma 2.1 we have

$$
0 \leq\left|u\left(\tau_{1}(t)\right)\right|^{\lambda}-\mu^{\lambda}(u) \leq\left|u\left(\tau_{1}(t)\right)-u\left(t_{0}\right)\right|^{\lambda} \leq v_{0}^{\lambda}\left\|u^{(n-1)}\right\|_{C_{\omega}}^{\lambda} \quad \text { for } t \in \mathbb{R} .
$$


If along with this we take into account inequalities (1.17), then from (3.14) we get

$$
\begin{aligned}
& \left(\int_{0}^{w} f(u)(s) d s\right) \operatorname{sgn}(\sigma u(0)) \geq h(\mu(u))-v_{0}^{\lambda} \int_{0}^{w}\left[p_{11}(s)\right]_{-} d s \times\left\|u^{(n-1)}\right\|_{C_{\omega}}^{\lambda} \\
& -\sum_{k=2}^{n} \int_{0}^{w} p_{1 k}(s) d s\left\|u^{(k-1)}\right\|_{C_{w}}-\int_{0}^{w} q(s) d s \quad \text { for } \mu(u)>0, \\
& \left|\int_{t}^{x} f(u)(s) d s\right| \leq \ell h(\mu(u))+v_{0}^{\lambda} \int_{0}^{w} p_{21}(s) d s\left\|u^{(n-1)}\right\|_{C_{\omega}}^{\lambda} \\
& +\sum_{k=2}^{n} \int_{0}^{w} p_{2 k}(s) d s\left\|u^{(k-1)}\right\|_{C_{\omega}}+\int_{0}^{w} q(s) d s \quad \text { for } 0 \leq t \leq x \leq \omega,
\end{aligned}
$$

where

$$
h(x)=x^{\lambda} \int_{0}^{w} p_{11}(s) d s
$$

and, in view of condition (1.18), $h$ is increasing and $h(x) \rightarrow+\infty$ as $x \rightarrow+\infty$.

If $\lambda<1$, then in view of (1.19) there exists $\varepsilon>0$ such that

$$
\varepsilon v_{0}^{\lambda} \int_{0}^{w}\left(\ell\left[p_{11}(s)\right]_{-}+p_{21}(s)\right) d s+\sum_{k=2}^{n} v_{k-1} \int_{0}^{w}\left(\ell p_{1 k}(s)+p_{2 k}(s)\right) d s<1
$$

Set

$$
\begin{gathered}
\varepsilon_{\lambda}=\varepsilon, \quad c_{0}=\int_{0}^{w} q(s) d s+\varepsilon^{-\lambda /(1-\lambda)} v_{0}^{\lambda}\left(\int_{0}^{w}\left(\left[p_{11}(s)\right]_{-}+p_{21}(s)\right) d s\right) \quad \text { for } \lambda<1, \\
\varepsilon_{\lambda}=1, \quad c_{0}=\int_{0}^{w} q(s) d s \quad \text { for } \lambda=1, \\
\ell_{1 k}=\int_{0}^{w} p_{1 k+1}(s) d s \quad(k=1, \ldots, n-2), \\
\ell_{1 n-1}=\varepsilon_{\curlywedge} v_{0}^{\lambda} \int_{0}^{w}\left[p_{11}(s)\right] d s+\int_{0}^{w} p_{1 n-1}(s) d s, \\
\ell_{2 k}=\int_{0}^{w} p_{2 k+1}(s) d s \quad(k=1, \ldots, n-2), \\
\ell_{2 n-1}=\varepsilon_{\curlywedge} v_{0}^{\lambda} \int_{0}^{w} p_{21}(s) d s+\int_{0}^{w} p_{2 n-1}(s) d s .
\end{gathered}
$$

Then by the Young inequality, inequalities (3.16) result in inequalities (1.11) and (1.12). On the other hand, the numbers $\ell_{i k}(i=1,2 ; k=1, \ldots, n-1)$ satisfy inequality (1.15) since for $\lambda<1$ (for $\lambda=1$ ) the functions $p_{i k}(i=1,2 ; k=1, \ldots, n)$ satisfy inequality (3.18) (inequality $(1.20))$.

Therefore, all the conditions of Theorem 1.1 are fulfilled which guarantee the existence of at least one $\omega$-periodic solution of (1.4). 
Proof of Corollary 1.4. Without loss of generality, it can be assumed that

$$
\int_{0}^{w} q(s) d s<c_{0}
$$

Then in view of (1.21), (1.22) from (3.14), inequalities (1.13) and (1.14) hold, where

$$
\ell_{k}=\int_{0}^{w} p_{k+1}(s) d s \quad(k=0, \ldots, n-1) .
$$

On the other hand, according to (1.23), the numbers $\ell_{k}(k=0, \ldots, n-1)$ satisfy (1.16). Therefore, all the conditions of Theorem 1.2 are fulfilled.

Proof of Theorem 1.5. For $v(t) \equiv 0$, inequalities (1.24) and (1.25) yield inequalities (1.11) and (1.12), where

$$
h(x)=\ell_{10} x, \quad c=\int_{0}^{w}|f(0)(s)| d s .
$$

Consequently, all the conditions of Theorem 1.1 are satisfied which guarantee the existence of at least one $\omega$-periodic solution of (1.3).

It remains to prove that (1.3) has no more than one $\omega$-periodic solution. Let $u_{1}$ and $u_{2}$ be arbitrary $\omega$-periodic solutions of (1.3) and

$$
u(t)=u_{2}(t)-u_{1}(t)
$$

If we assume that $\mu(u)>0$, then from (1.24) we find

$$
\ell_{10} \mu(u) \leq \sum_{k=1}^{n-1} \ell_{1 k}\left\|u^{(k)}\right\|_{C_{\omega}}
$$

It is obvious that this inequality is valid also for $\mu(u)=0$. Due to (3.24), from (1.25) it follows

$$
\left\|u^{(n-1)}\right\|_{C_{\omega}} \leq \max \left\{\left|\int_{t}^{x}\left(f\left(u_{1}+u\right)(s)-f\left(u_{1}\right)(s)\right) d s\right|: 0 \leq t \leq x \leq \omega\right\} \leq \sum_{k=1}^{n-1} \ell_{k}\left\|u^{(k)}\right\|_{C_{\omega^{\prime}}}
$$

where $\ell_{k}=\ell \ell_{1 k}+\ell_{2 k}(k=1, \ldots, n-1)$. Moreover, in view of (1.15) the numbers $\ell_{k}(k=$ $1, \ldots, n-1)$ satisfy inequality (2.17). On the other hand, by Lemma 2.1 from (3.24) we have

$$
\ell_{10} \mu(u) \leq\left(\sum_{k=1}^{n-1} \ell_{1 k} v_{k}\right)\left\|u^{(n-1)}\right\|_{C_{\omega}} .
$$

Consequently, inequalities (2.16) are satisfied, where

$$
\varphi(x)=\left(\sum_{k=1}^{n-1} \ell_{10}^{-1} \ell_{1 k} v_{k}\right) x, \quad c_{0}=0 .
$$

If now we apply Lemma 2.3, then it becomes evident that $u(t) \equiv 0$, that is, $u_{1}(t) \equiv u_{2}(t)$. 
Proof of Corollary 1.7. To prove the corollary, it is sufficient to state that the operator $f$, given by equality (3.14), satisfies the conditions of Theorem 1.5.

Let $u$ and $v \in C_{\omega}^{n-1}$. Then by virtue of conditions (1.29), and Lemma 2.1, from (3.14), we obtain inequalities (1.24) and (1.25), where

$$
\begin{array}{lll}
\ell_{1 k}=\int_{0}^{w} p_{1 k+1}(s) d s & (k=0, \ldots, n-2), & \ell_{1 n-1}=v_{0} \int_{0}^{w}\left[p_{11}(s)\right]_{-} d s+\int_{0}^{w} p_{1 n-1}(s) d s, \\
\ell_{2 k}=\int_{0}^{w} p_{2 k+1}(s) d s & (k=0, \ldots, n-2), & \ell_{2 n-1}=v_{0} \int_{0}^{w}\left[p_{21}(s)\right]_{-} d s+\int_{0}^{w} p_{2 n-1}(s) d s .
\end{array}
$$

On the other hand, in view of (1.18) and (1.20), $\ell_{10}>0$ and condition (1.15) holds, where $\ell=\ell_{20} / \ell_{10}$.

Proof of Theorem 1.6. For $v(t) \equiv 0,(1.26)-(1.28)$ yield conditions (1.13), (1.14), and (1.16), where

$$
c_{0}=\int_{0}^{w}|f(0)(s)| d s, \quad \ell_{k}=0 \quad(k=1, \ldots, n-1) .
$$

Consequently, all the conditions of Theorem 1.2 are satisfied which guarantee the existence of at least one $\omega$-periodic solution of (1.3).

Suppose now that $u_{1}$ and $u_{2}$ are arbitrary $\omega$-periodic solutions of $(1.3)$ and $u(t)=u_{2}(t)-$ $u_{1}(t)$. If we assume that $\mu(u)>0$, then in view of (1.26) we obtain the contradiction

$$
0=\left(\int_{0}^{w} u^{(n)}(s) d s\right) \operatorname{sgn}(\sigma u(0))=\left(\int_{0}^{w}\left(f\left(u_{1}+u\right)(s)-f\left(u_{1}\right)(s)\right) d s\right) \operatorname{sgn}(\sigma u(0))>0 .
$$

Thus, it is proved that $\mu(u)=0$.

On the other hand, (1.27) implies

$$
\left\|u^{(n-1)}\right\|_{C_{\omega}} \leq \ell_{0}\|u\|_{C_{\omega}} .
$$

Therefore, inequalities (2.22) are satisfied, where $c_{0}=0, \ell_{k}=0(k=1, \ldots, n-1)$, and $\ell_{0} v_{0}<1$. Hence by Lemma 2.4 it follows that $u(t) \equiv 0$, that is, $u_{1}(t) \equiv u_{2}(t)$.

Proof of Corollary 1.8. For any $u \in C_{\omega}^{n-1}$, we set

$$
f(u)(t)=g(u)(t)
$$

Then conditions (1.33) and (1.34) imply conditions (1.26)-(1.28), where $\ell_{0}=\int_{0}^{\omega} p(s) d s$. Consequently, the operator $f$ satisfies all the conditions of Theorem 1.6.

\section{Examples}

From the main results of the present paper new (and optimal in some sense) sufficient conditions for the existence of periodic solutions of linear and sublinear differential equations with 
deviated arguments and differential equations with bounded right-hand sides follow. To illustrate the above mentioned, let us consider the differential equations

$$
\begin{gathered}
u^{(n)}(t)=\sum_{k=1}^{n} g_{k}(t)\left|u^{(k-1)}\left(\tau_{k}(t)\right)\right|^{\lambda_{k}} \operatorname{sgn}\left(u^{(k-1)}\left(\tau_{k}(t)\right)\right)+g_{0}(t), \\
u^{(n)}(t)=\sum_{k=1}^{n} g_{k}(t)\left|u^{(k-1)}\left(\tau_{k}(t)\right)\right|^{\lambda_{0 k}}\left(1+\left|u\left(\tau_{1}(t)\right)\right|\right)^{-\lambda_{k}} u\left(\tau_{1}(t)\right)+g_{0}(t), \\
u^{(n)}(t)=\sum_{k=1}^{n} g_{k}(t) u^{(k-1)}\left(\tau_{k}(t)\right)+g_{0}(t), \\
u^{(n)}(t)=\sum_{k=1}^{m} g_{0 k}(t) \frac{|u(\tau(t))|^{\lambda_{k}}}{1+|u(\tau(t))|^{\lambda_{k}}} \operatorname{sgn} u(\tau(t))+g_{0}(t),
\end{gathered}
$$

where $m$ is a natural number, $\lambda_{k}>0(k=1, \ldots, m), \lambda_{0 k} \geq 0(k=1, \ldots, n)$ are constants, $g_{k} \in L_{\omega}(k=0, \ldots, n), g_{0 k} \in L_{\omega}(k=1, \ldots, m)$, while $\tau_{k}: \mathbb{R} \rightarrow \mathbb{R}(k=1, \ldots, n)$ and $\tau: \mathbb{R} \rightarrow \mathbb{R}$ are measurable on every finite interval functions satisfying, respectively, conditions (1.7) and (1.32).

Corollaries 1.3 and 1.4 imply the following corollaries.

Corollary 4.1. Let

$$
\int_{0}^{w} g_{1}(s) d s \neq 0
$$

and either

$$
0<\lambda_{k}<1 \quad(k=1, \ldots, n)
$$

or

$$
0<\lambda_{1}<1, \quad 0<\lambda_{k} \leq 1 \quad(k=2, \ldots, n), \quad \sum_{k=2}^{n} v_{k-1} \int_{0}^{w}\left|g_{2 k}(s)\right| d s<(1+\ell)^{-1}
$$

where

$$
\ell=\frac{\int_{0}^{1}\left|g_{1}(s)\right| d s}{\left|\int_{0}^{1} g_{1}(s) d s\right|}
$$

Then (4.1) has at least one w-periodic solution.

Corollary 4.2. Let

$$
\int_{0}^{w} g_{0}(s) d s=0
$$

and there exists a constant $\sigma \in\{-1,1\}$ such that

$$
\sigma g_{k}(t) \geq 0 \quad \text { for } t \in \mathbb{R}(k=1, \ldots, n) .
$$

Let, moreover, either

$$
0 \leq \lambda_{01}<\lambda_{1}, \quad 0 \leq \lambda_{0 k}<1, \quad \lambda_{k} \geq 1 \quad(k=2, \ldots, n),
$$


or

$$
0 \leq \lambda_{01} \leq \lambda_{1}, \quad 0 \leq \lambda_{0 k} \leq 1, \quad \lambda_{k} \geq 1 \quad(k=2, \ldots, n),
$$

and

$$
\sum_{k=1}^{n} v_{k-1} \int_{0}^{w}\left|g_{k}(s)\right| d s<1 .
$$

Then (4.2) has at least one w-periodic solution.

Remark 4.3. If

$$
g_{k}(t) \equiv 0 \quad(k=1, \ldots, n), \quad \int_{0}^{w} g_{0}(s) d s \not \equiv 0,
$$

then (4.1) has no w-periodic solution. Consequently, conditions (1.18) and (4.5) in Corollaries 1.3 and 4.1 are essential and they cannot be omitted.

Remark 4.4. If

$$
\begin{gathered}
\lambda_{0 k}=0, \quad \lambda_{k} \geq 1 \quad(k=1, \ldots, n), \quad \sum_{k=1}^{n} \int_{0}^{w}\left|g_{k}(s)\right| d s \leq \varepsilon, \\
\\
\left|\int_{0}^{w} g_{0}(s) d s\right|=\varepsilon,
\end{gathered}
$$

where $\varepsilon>0$, then (4.2) has no $w$-periodic solution. Consequently, conditions (1.21) and (4.9) in Corollaries 1.4 and 4.2 cannot be replaced by condition (4.16) no matter how small $\varepsilon$ would be.

Corollary 1.7 yields the following.

Corollary 4.5. Let there exist a constant $\sigma \in\{-1,1\}$ such that

$$
\begin{gathered}
\sigma \int_{0}^{w} g_{1}(s) d s>0 \\
v_{0} \int_{0}^{w}\left(\ell\left[\sigma g_{1}(s)\right]_{-}+\left|g_{1}(s)\right|\right) d s+(1+\ell) \sum_{k=2}^{n} v_{k-1} \int_{0}^{w}\left|g_{k}(s)\right| d s<1,
\end{gathered}
$$

where $\ell$ is a number given by equality (4.8). Then (4.3) has one and only one w-periodic solution.

Suppose

$$
\eta(\lambda)=\frac{1}{4 \lambda}(\lambda-1)^{(\lambda-1) / \lambda}(\lambda+1)^{(\lambda+1) / \lambda} \quad \text { for } \lambda>1, \quad \eta(\lambda)=1 \quad \text { for } \lambda=1 .
$$

Theorem 1.6 results in the following.

Corollary 4.6. Let there exist a constant $\sigma \in\{-1,1\}$ such that

$$
\sigma g_{0 k}(t) \geq 0 \quad \text { for } t \in \mathbb{R}(k=1, \ldots, m)
$$


If, moreover,

$$
\begin{gathered}
\sum_{k=1}^{m} \int_{0}^{w}\left|g_{0 k}(s)\right| d s>0, \\
\sum_{k=1}^{m} \eta\left(\lambda_{k}\right) \int_{0}^{w}\left|g_{0 k}(s)\right| d s<\frac{1}{v_{0}},
\end{gathered}
$$

and equality (4.9) holds, then (4.4) has one and only one w-periodic solution.

Remark 4.7. If $g_{0 k}(t) \equiv 0(k=1, \ldots, m)$ and equality (4.9) is fulfilled, then (4.4) has an infinite set of $\omega$-periodic solutions. Consequently, the strict inequality (1.26) (the strict inequality (4.20)) in Theorem 1.6 (in Corollary 4.6) cannot be replaced by nonstrict one.

\section{Acknowledgments}

The first two authors are supported by the Georgian National Science Foundation (Grant no. GNSF/ST06/3-002), and the third author is supported by the Ministry of Education of Czech Republic (Research Project no. MSM0021622409).

\section{References}

[1] A. Lasota and Z. Opial, "Sur les solutions périodiques des équations diffèrentielles ordinaires," Annales Polonici Mathematici, vol. 16, no. 1, pp. 69-94, 1964.

[2] M. A. Krasnosel'skij, The Operator of Translation along the Trajectories of Differential Equations, Nauka, Moscow, Russia, 1966.

[3] S. R. Bernfeld and V. Lakshmikantham, An Introduction to Nonlinear Boundary Value Problems, vol. 10 of Mathematics in Science and Engineering, Academic Press, New York, NY, USA, 1974.

[4] R. E. Gaines and J. L. Mawhin, Coincidence Degree, and Nonlinear Differential Equations, vol. 56 of Lecture Notes in Mathematics, Springer, Berlin, Germany, 1977.

[5] L. A. Kipnis, "On periodic solution of higher order nonlinear differential equations," Prikladnaya Matematika i Mekhanika, vol. 41, no. 2, pp. 362-365, 1977, (Russian).

[6] F. W. Bates and J. R. Ward Jr, "Periodic solutions of higher order systems," Pacific Journal of Mathematics, vol. 84 , no. 2, pp. 275-282, 1979.

[7] G. T. Gegelia, "On boundary value problems of periodic type for ordinary odd order differential equations," Archivum Mathematicum, vol. 20, no. 4, pp. 195-204, 1984.

[8] I. Kiguradze, "Bounded and periodic solutions of higher-order linear differential equations," Matematicheskie Zametki, vol. 37, no. 1, pp. 48-62, 138, 1985, English translation in Mathematical Notes, vol. 37, no.1, pp. 28-36, 1985, (Russian).

[9] G. T. Gegelia, "On bounded and periodic solutions of even-order nonlinear ordinary differential equations," Differentsial'nye Uravneniya, vol. 22, no. 3, pp. 390-396, 547, 1986, (Russian).

[10] G. T. Gegelia, "On periodic solutions of ordinary differential equations," in Qualitative Theory of Differential Equations (Szeged, 1988), vol. 53 of Colloquia Mathematica Societatis János Bolyai, pp. 211-217, North-Holland, Amsterdam, The Netherlands, 1990.

[11] Yu. V. Trubnikov and A. I. Perov, Differential Equations with Monotone Nonlinearities, Nauka i Tekhnika, Minsk, Russia, 1986.

[12] I. Kiguradze, Initial and Boundary Value Problems for Systems of Ordinary Differential Equations. I, Metsniereba, Tbilisi, Russia, 1997.

[13] I. Kiguradze and T. Kusano, “On periodic solutions of higher-order nonautonomous ordinary differential equations," Differentsial'nye Uravneniya, vol. 35, no. 1, pp. 72-78, 142, 1999, English translation in Differential Equations, vol. 35, no.1, pp. 71-77, 1999, (Russian).

[14] I. Kiguradze, "On periodic solutions of $n$-th order ordinary differential equations," Nonlinear Analysis: Theory, Methods E Applications, vol. 40, no. 1-8, pp. 309-321, 2000. 
[15] I. Kiguradze and T. Kusano, "On conditions for the existence and uniqueness of a periodic solution of nonautonomous differential equations," Differentsial'nye Uravneniya, vol. 36, no. 10, pp. 1301-1306, 1436, 2000, English translation in Differential Equations, vol. 36, no.10, pp. 1436-1442, 2000, (Russian).

[16] I. Kiguradze and T. Kusano, "On periodic solutions of even-order ordinary differential equations," Annali di Matematica Pura ed Applicata, vol. 180, no. 3, pp. 285-301, 2001.

[17] S. R. Baslandze and I. Kiguradze, "On the unique solvability of a periodic boundary value problem for third-order linear differential equations," Differentsial'nye Uravneniya, vol. 42, no. 2, pp. 153-158, 285, 2006, English translation in Differential Equations, vol. 42, no.2, pp. 165-171, 2006, (Russian).

[18] M. Schechter, "Periodic solutions of second-order nonautonomous dynamical systems," Boundary Value Problems, vol. 2006, Article ID 25104, 9 pages, 2006.

[19] I. Kiguradze and B. Půža, "On periodic solutions of nonlinear functional differential equations," Georgian Mathematical Journal, vol. 6, no. 1, pp. 47-66, 1999.

[20] I. Kiguradze and B. Půža, "On periodic solutions of systems of differential equations with deviating arguments," Nonlinear Analysis: Theory, Methods \& Applications, vol. 42, no. 2, pp. 229-242, 2000.

[21] R. Hakl, A. Lomtatidze, and B. Půža, "On periodic solutions of first order nonlinear functional differential equations of non-Volterra's type," Memoirs on Differential Equations and Mathematical Physics, vol. 24, pp. 83-105, 2001.

[22] R. Hakl, A. Lomtatidze, and B. Půža, "On periodic solutions of first order linear functional differential equations," Nonlinear Analysis: Theory, Methods \& Applications, vol. 49, no. 7, pp. 929-945, 2002.

[23] R. Hakl, A. Lomtatidze, and J. Šremr, "On a periodic type boundary-value problem for first order linear functional differential equations," Nonlinear Oscillations, vol. 5, no. 3, pp. 416-433, 2002.

[24] R. Hakl, A. Lomtatidze, and J. Šremr, "On a periodic type boundary value problem for first order nonlinear functional differential equations," Nonlinear Analysis: Theory, Methods \& Applications, vol. 51, no. 3, pp. 425-447, 2002.

[25] R. Hakl, A. Lomtatidze, and J. Šremr, “On constant sign solutions of a periodic type boundary value problem for first order scalar functional differential equations," Memoirs on Differential Equations and Mathematical Physics, vol. 26, pp. 65-90, 2002.

[26] R. Hakl, A. Lomtatidze, and J. Šremr, Some Boundary Value Problems for First Order Scalar Functional Differential Equations, Masaryk University, Brno, Czech Republic, 2002.

[27] I. Kiguradze and B. Půža, Boundary Value Problems for Systems of Linear Functional Differential Equations, vol. 12 of Folia Facultatis Scientiarium Naturalium Universitatis Masarykianae Brunensis. Mathematica, Masaryk University, Brno, Czech Republic, 2003.

[28] S. Mukhigulashvili, "On a periodic boundary value problem for second-order linear functional differential equations," Boundary Value Problems, no. 3, pp. 247-261, 2005.

[29] S. Mukhigulashvili, "On the solvability of a periodic problem for second-order nonlinear functionaldifferential equations," Differentsial'nye Uravneniya, vol. 42, no. 3, pp. 356-365, 430, 2006, (Russian).

[30] S. Mukhigulashvili, "On periodic solutions of second order functional differential equations," Italian Journal of Pure and Applied Mathematics, no. 20, pp. 29-50, 2006.

[31] S. Mukhigulashvili, "On a periodic boundary value problem for cyclic feedback type linear functional differential systems," Archiv der Mathematik, vol. 87, no. 3, pp. 255-260, 2006.

[32] G. H. Hardy, J. E. Littlewood, and G. Pólya, Inequalities, Cambridge University Press, Cambridge, UK, 2nd edition, 1952.

[33] I. Kiguradze and B. Půža, "On boundary value problems for functional differential equations," Memoirs on Differential Equations and Mathematical Physics, vol. 12, pp. 106-113, 1997. 